\title{
Connaître ? Agir ? Interagir ?
}

Plusieurs textes de ce numéro nous interpellent à propos de la connaissance, de ses processus de construction, des cadres conceptuels qui l'organisent, de sa formalisation par les modèles, de son appropriation par le sens commun ou l'action publique...

Les processus de connaissance sont à l'arrière-plan du travail de recherche. Pour nous, ils sont également à la base des - et, pourrait-on dire plus fortement encore, en jeu dans les - tentatives de dépassement des démarches disciplinaires auxquelles nous sommes si attachés à NSS . Mais s'en tenir à une posture interdisciplinaire fondée sur une confrontation des points de vue disciplinaires fait l'impasse sur les limites intrinsèques à toute démarche de connaissance. Une approche interdisciplinaire peut être ainsi victime d'une certaine cécité et elle doit pour le moins être consciente de ses limites. La transdisciplinarité, au sens d'une intégration et d'un dépassement des disciplines, est-elle une alternative qui peut être mise en pratique (voire qui doit l'être) ou simplement un cadre de référence ultime, une sorte de " point oméga » permettant de mieux comprendre les ressorts cognitifs qui sont à l'arrière-plan de l'interdisciplinarité, afin de pratiquer celle-ci de façon plus réflexive?

Toute connaissance procède de la perception que nous avons du monde, nous rappellent Jean-Pierre Bréchet et Gérard Gigand dans leur article, rendant ainsi explicite que nous construisons la connaissance sur l'inconnaissabilité radicale qu'engage notre présence au monde. Ils proposent une ingénierie représentationnelle en recourant à un appareillage intellectuel basé sur un schéma ternaire (incomplétude, autoréférence et indétermination) pour comprendre et traiter la réalité phénoménologique.

C'est effectivement une posture essentielle, car le monde qui nous entoure n'existe que par la façon dont nous le percevons et dont nous concevons nos interactions avec les êtres et les objets qui le composent. Et ce sont ces interactions qui construisent la dynamique de nos perceptions de l'existant. Cela est particulièrement vrai dans nos relations avec les autres êtres vivants, humains certes, mais également avec notre environnement bio- physique au sein duquel nous agissons, vivons, pensons ${ }^{1}$. Nous ne percevons ce monde qu'à partir de ce que nous sommes, c'est-à-dire des interactions, des relations que nous sommes capables de construire avec les betes, les gens, les choses qui le constituent, et à partir des intentions avec lesquelles nous entrons en relation. Une telle posture conduit à revoir les distinctions usuelles entre action et connaissance, comme entre "technê » et «epistêmê ». Et si, justement, il n'y avait pas de différence substantielle entre agir et connaître, à partir du moment où ils procèdent l'un et l'autre des interactions que génère toute praxis, celle-ci étant une expérience singulière de notre rapport aux autres, au monde biophysique et aux transformations ainsi engendrées ? C'est d'ailleurs ce à quoi nous invitent également ceux qui distinguent ${ }^{2}$ les connaissances établies (knowledge), enseignables et transmissibles, et les connaissances en train de se produire au cours de l'action (knowing), pour les appeler à interagir dans le cadre d'une danse créative (generative dance) à l'origine de l'inédit, du nouveau, du changement... Ce sont dans les interactions que cette dualité émerge, et plus particulièrement lors des échanges réitérés entre les différentes connaissances, par exemple les connaissances scientifiques du chercheur, les connaissances techniques des praticiens et les savoirs en action des responsables administratifs ou politiques en charge de la gestion d'une situation ; cette dualité émerge également lors des élaborations collectives et lors des échanges de points de vue entre ces différents acteurs, chercheurs et non-chercheurs. Ce sont toutes ces interactions qui engendrent des conditions nouvelles et donc une situation nouvelle. Dans son texte publié dans ce numéro, Marc Mormont nous rappelle que John Dewey va même jusqu'à préférer parler de «trans-action» afin de dépasser la notion d'interaction

\footnotetext{
1 Voir, par exemple, Hubert, B., 2004. Pour une écologie de l'action. Savoir agir, apprendre, connaître, Paris, Éditions Arguments.

2 Comme Cook, S.D.N., Brown, J.S., 1999. Bridging epistemologies: the generative dance between organizational knowledge and organizational knowing, Organization Science, 10, 4, 381-400.
} 
entre des entités préexistantes pour renforcer l'idée d'un flux d'échanges permanent, transformant lui-même les diverses entités ainsi reliées.

Nous retrouvons ici le dépassement ${ }^{3}$ de l'opposition entre empirisme et idéalisme afin de concevoir de façon systémique à la fois l'objet à penser et la théorie construite de cet existant, et afin d'inventer les concepts et les pratiques qui induisent le changement, élaborent et transforment les normes et donc les problèmes à l'origine de cette mise en dynamique, si possible collective.

Et nous sommes bien alors engagés dans une dynamique cognitive inaboutie... Nous avons compris aujourd'hui que l'accumulation des connaissances ne diminue que rarement l'incertitude, mais l'accroît du fait de la mise en lumière de nouvelles interdépendances. C'est ce qui, entre autres, nous autorise à qualifier de « complexes" certaines situations dans lesquelles nous nous engageons, ainsi que les objets que nous concevons pour y agir, notamment en termes de production de connaissances. Il ne s'agit plus de réduire ou d'éliminer l'incertitude, mais de la gérer. Traiter ces situations relève alors du partage de connaissances, de processus $d^{\prime}$ apprentissage et d'explorations qui ouvrent de nouveaux espaces à l'action, en modifiant à la fois la dimension cognitive (la compréhension de la situation) et la dimension pratique (les actions menées) : la situation change parce que nous changeons avec elle ${ }^{4} \ldots$

N'est-ce pas alors aller au bout des approches dites «systèmes » en distinguant ${ }^{5}$ une posture "ontologique » (les systèmes sont dans le monde et il suffit de savoir les voir afin de prendre en compte la complexité qu'on accorde au monde) et une posture "épistémologique » (un outillage heuristique qui consiste à décider de voir le monde comme faisant système, nous-mêmes compris). «Nous ne raisonnons que sur des modèles » nous dit Paul Valéry, et d'ailleurs, en modélisation systémique, la tâche la plus importante du modélisateur n'est pas de résoudre le problème posé, mais de résoudre $\mathrm{d}^{\prime}$ abord le problème qui consiste à poser le problème ${ }^{6}$. La recherche, n'est-ce pas avant tout un exercice de «reflective learning in the literal sense... it is the thinking about thinking, doubting about doubting, learning about learning, and (hopefully) knowing about knowing ${ }^{7} »$. De ce point de vue, le cadrage du problème à formuler afin de le traiter en situation détermine comment nous (individuellement ou collectivement) allons penser et agir dans cette situation d'une manière systémique, c'est-à-dire en distinguant un ensemble cohérent dans son contexte, sans préjuger d'un domaine disciplinaire qui serait à privilégier.

Une telle approche, fondée sur une praxis, nous permet de relativiser les débats d'écoles et de disciplines en nous appelant à la plus grande réflexivité sur la production de connaissances scientifiques et les manières dont elles imprègnent le monde commun qui en fait usage...

Bernard Hubert, Catherine Aubertin, Jean-Paul Billaud

\footnotetext{
3 Auquel nous invite Jean-Pierre Darré (Darré, J.-P., 1999. La production de connaissances pour l'action. Arguments contre le racisme de l'intelligence, Paris, Éditions MSH/Inra).

4 Hatchuel, A., Weil, B., 2009. C-K design theory: an advanced formulation, Research in Engineering Design, 19, 4, 181-192.

5 Sans les opposer car elles peuvent se combiner si elles sont assumées comme telles, formant là aussi une dualité ainsi que le propose Ray Ison (Ison, R., 2010. Systems practice: how to act in a climate-change world, Springer).

6 Pour suivre Jean-Louis Le Moigne (Le Moigne, J.-L., 1994 [1 ${ }^{\mathrm{re}}$ éd : 1977]. La théorie du système général. Théorie de la modélisation, Paris, PUF ; publiée en ligne en 2006 dans la collection «Les Classiques du Réseau Intelligence de la Complexité », http:// www.mcxapc.org/inserts/ouvrages/0609tsgtm.pdf).

7 Churchman, C.W., 1972. The design of inquiring systems. Basic concepts of systems and organizations, New York, Basic Books.
} 